\title{
Towards Anthropomorphic Robot Thereminist
}

\author{
Yan Wu, Polake Kuvinichkul, Peter Y.K. Cheung, Yiannis Demiris
}

\begin{abstract}
Theremin is an electronic musical instrument considered to be the most difficult to play which requires the player's hands to have high precision and stability as any position change within proximity of the instrument's antennae can make a difference to the pitch or volume. In a different direction to previous developments of Theremin playing robots, we propose a Humanoid Thereminist System that goes beyond using only one degree of freedom which will open up the possibility for robot to acquire more complex skills, such as aerial fingering and include musical expressions in playing the Theremin. The proposed system consists of two phases, namely calibration phase and playing phase which can be executed independently. During the playing phase, the System takes input from a MIDI file and performs path planning using a combination of minimum energy strategy in joint space and feedback error correction for next playing note. Three experiments have been conducted to evaluate the developed system quantitatively and qualitatively by playing a selection of music files. The experiments have demonstrated that the proposed system can effectively utilise multiple degrees of freedoms while maintaining minimum pitch error margins.
\end{abstract}

Index Terms - robotic Thereminist, music playing robot, aerial fingering, humanoid, iCub

\section{INTRODUCTION}

The recent development of humanoid robots indicates the growing interest in making robots to improve the quality of our daily life. To many people, music forms an integral part of their life. Thus, it is natural to expect that should robots become our companions, they must also possess the skills to interact with us in daily environments through this universal language of mankind [1]. There has been some research effort focusing on developing various musical playing robots, such as robotic percussionist [2], pianist [3], flutist [4] and violinist [5]. Many of these platforms have adopted musical instruments that are insensitive to environmental fluctuations and have fixed physical contacts.

However, little work has been done on Theremin, one of the most difficult instruments to play [6]. Named after a Russian physicist Léon Theremin, the Theremin is an electronic musical instrument invented in the 1920s. It is a unique type of musical instruments which requires no physical contact to play music. As shown in Fig. 1, a musical tone can be generated by interactions that change the capacitance of the antennae. This makes it very sensitive to changes in the surrounding electromagnetic field which requires the Theremin to be calibrated each time when it is

Yan Wu, Polake Kuvinichkul, Peter Y.K. Cheung and Yiannis Demiris are with Department of Electrical \& Electronic Engineering, Imperial College London, United Kingdom \{yan.wu08, polake.kuvinichkul06, p.cheung, y.demiris\}@imperial.ac.uk played. As any slight change in position within the proximity of the antennae can make a difference to the pitch or volume, the player's hands must have high precision and stability. Also the non-linearity of the aerial capacitance and absence of visual reference point for each note increase the learning difficulty for an untrained player.

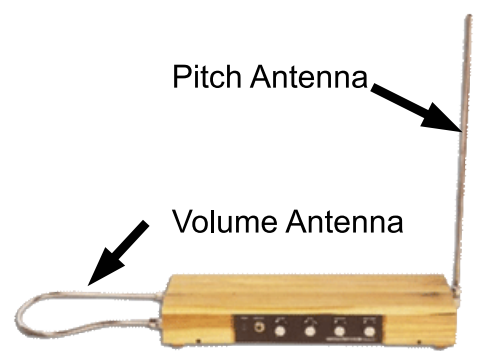

Fig. 1: Theremin has 2 antennae controlling pitch \& volume respectively.

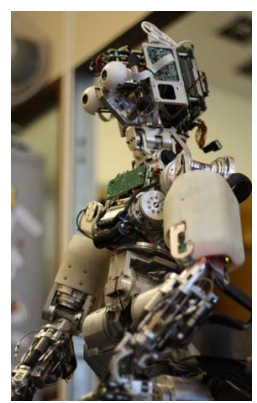

Fig. 2: The iCub

A Theremin-playing robot was first developed by Alford et al [7]. In their work, a thorough sweeping of a one-degreeof-freedom(DoF) arm is used to search and store the arm positions of corresponding musical notes. This work assumes environmental invariance and hence requires no feedback control during performance. To speed up the calibration process and adapt to environmental changes, Mizumoto et al recently proposed a design to estimate the parameters of their Theremin pitch model [8]. Although this approach typically requires 12 samples for good model estimation, it assumes that the movement of the arm is radial to the pitch antenna. This demands for the robot to have advanced inverse kinematics algorithm to eliminate incorrect transient pitches during arm movements. Furthermore, the pitch model is valid for a limited range of objects and materials interacting with the antenna.

In terms of movement planning, both works use the "coarse-to-fine" approach with some combination of feedforward and feedback controllers. While the feed-forward controller is used to move to the proximity of the intended note quickly, the feedback controller is used to move to the exact note position. In [7], feedback control is only introduced in the calibration phase with a non-linear proportional controller. In [8], a proportional-integral (PI) controller is deployed in the playing phase. As melody is timecritical, feedback control within a particular note might cause irregularity in the musical rhythm. Moreover, to improve performance, expert Thereminists are trained to acquire a technique called "Aerial Fingering" which uses fingers to 
change tones quickly and reliably. Comparing this skill to the current robotic Thereminists which are constrained to move in one degree of freedom ( $\mathrm{DoF}$ ), the potential to augment skill acquisition with an anthropomorphic robot arm, i.e. using multiple DoFs, is yet to be explored.

In this paper, we propose a Humanoid Thereminist System which gives a Humanoid robot the possibility to mimic playing skills of a human Thereminist. This is a two-phase system which comprises a calibration phase and a playing phase. It has two novel features: a) The system make use of Dynamic Programming [9] approach and minimum energy strategy to enable multiple DoFs of an anthropomorphic robot arm to be utilised in playing the Theremin. b) It accounts for environmental changes during the playing phase with a forward model to fine tune the upcoming notes. This trade-off between absolute pitch and rhythm is supported by studies [10], [11] showing ordinary humans are more sensitive to tempo than pitch errors (even at very young age). The following sections of this paper will present some preliminary findings that are essential to our approach followed by a detailed description of our Humanoid Thereminist System. We will also discuss three experiments designed to evaluate the System both quantitatively and qualitatively using the iCub ${ }^{1}$ Humanoid Robot (Fig. 2).

\section{PRELIMINARIES}

In this section, we describe two topics of preliminary investigation that serve as building blocks of our Humanoid Thereminist System.

\section{A. Pitch Estimation Methods}

Pitch estimation serves as a form of feedback to the system on where a particular note is and how accurately the note can be played. In playing phase, if pitch estimation is required, it also has a critical role in determining how fast a feedback action can be. Pitch can be estimated either in time-domain or frequency-domain. In [7], Fast Fourier Transform (FFT) [12], a frequency-domain estimation method is used while [8] uses a time-domain estimation method called Auto-correlation (AC) [13]. Considering that the Theremin has a low-pass filter at its output, a faster time-domain-based algorithm, the Zero-Crossing Rate (ZCR) [14] can also be used.

Since pitch estimation affects both pitch and tempo in robotic music playing, we conducted a series of experiments to select the best algorithm to be used by our system based on maximum error and average time-taken for each of these methods over a range of 3 musical Octaves ( 36 notes) played from a synthetic Theremin audio input. The results are summarised in Table I which shows that FFT has its Average Time-taken in the order of that of ZCR while having the lowest Max Error. Although more complex variant of ZCR are available to reduce false detection rate, these methods will nonetheless increase the computational complexity and hence time-taken. This makes FFT stand out as the best choice for implementation.

\footnotetext{
${ }^{1}$ Developed by the RobotCub Consortium (www.robotcub.org)

${ }^{2}$ Open-source library FFTW (www.fftw.org) is used.
}

TABLE I: Performance of pitch estimation methods over 3 Octaves played using synthetic Theremin.

\begin{tabular}{l||c|c|c}
\hline & ZCR & AC & FFT $^{2}$ \\
\hline Max Error (\%) & 151.40 & 2.04 & 0.17 \\
\hline Ave Time-taken (ms) & 0.103 & 38.546 & 0.594 \\
\hline
\end{tabular}

\section{B. Non-parametric Interpolation Model}

In [8], the authors describe a non-parametric Theremin pitch model for invariant environment. In this model, a linear interpolation method is used to find the arm position $\mathbf{x}$ of the required pitch frequency $f$, if $f$ lies within the range of the calibrated frequencies, and it is simply given as:

$$
\mathbf{x}=\mathbf{x}_{l}+\left(\mathbf{x}_{h}-\mathbf{x}_{l}\right) \frac{f-f_{l}}{f_{h}-f_{l}}
$$

where $f_{l}$ denotes the frequency of a note immediately lower than $f$ with its position $\mathbf{x}_{l}$ and $f_{h}$ denotes the frequency of a note immediately higher with position $\mathbf{x}_{h}$.

However, the pitch characteristics of the Theremin is known to be highly non-linear and determined by the capacitance formed between the antenna and the arm. (1) will introduce satisfactory amount of pitch error only if the upper and lower frequencies are very close to each other. Despite the characteristics of the variable capacitance, it is surprising to discover that great linearity is exhibited between musical note number $p$ and arm angle $\theta$, where $p$ is given by (2) and $\theta$ is the angle shown in Fig. 3.

$$
p=69+12 \times \log _{2} \frac{f}{440}
$$

In this experiment, we only allow the right arm of the robot to rotate in the shoulder yaw axis. Various distances between the Theremin and the iCub have been experimented. Fig. 4 shows an instance of the results where the distance is $50 \mathrm{~cm}$.
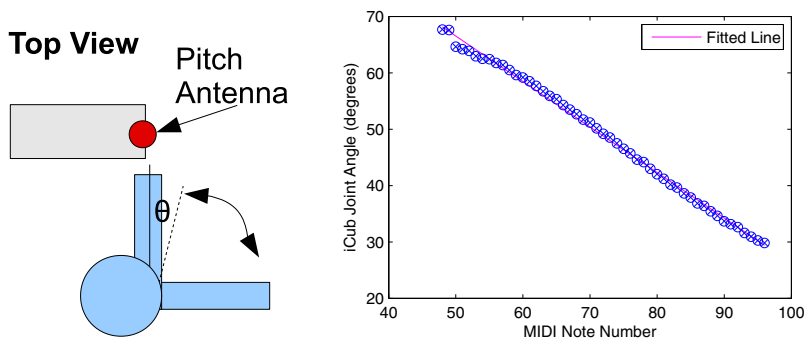

Fig. 3: Setup of the 1 Fig. 4: Graph of Right Arm Angle $\theta$ v.s. DoF Experiment MIDI Note No. $p$ for the 1 DoF Exp.

The line-of-best-fit in this graph yields a correlation coefficient $\left(R^{2}\right)$ of 0.997 which suggests a very high linear relationship between $\theta$ and $p$. Thus, we can derive an adapted interpolation model for an arm where the axis of rotation is parallel to the pitch antenna:

$$
\theta=\theta_{l}+\left(\theta_{h}-\theta_{l}\right) \frac{p-p_{l}}{p_{h}-p_{l}}
$$


where $p_{l}$ denotes the note number of a note immediately smaller than $p$ with its arm angle $\theta_{l}$ and $p_{h}$ denotes the note number of a note immediately greater with angle $\theta_{h}$.

\section{HUMANOID THEREMINIST SYSTEM}

Fig. 5 shows the block diagram of our Humanoid Thereminist System. This system consists of two phases and an independent module, the calibration phase, the playing phase and the pitch/volume analyser. The playing phase can be run independently from the calibration phase so long as the calibrated configurations have been stored properly. In the sections below, for simplicity of discussion, we shall assume the left arm controls volume while the right arm controls pitch.

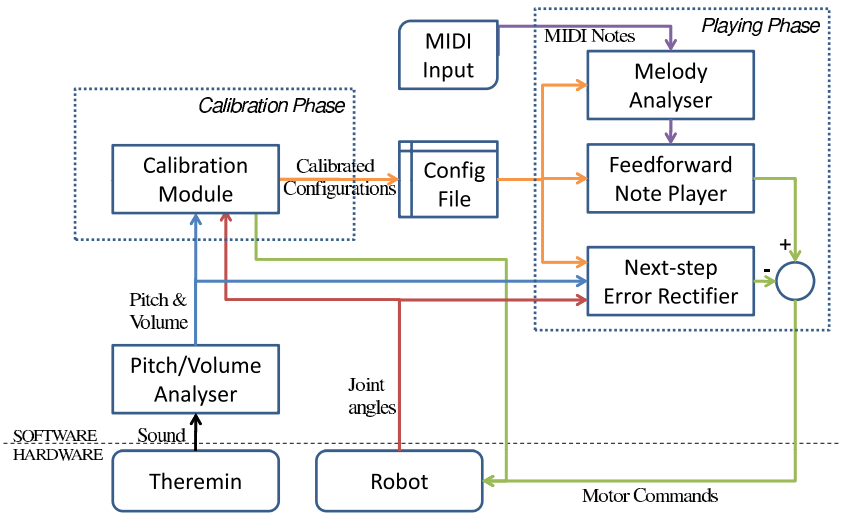

Fig. 5: The Humanoid Thereminist System

\section{A. Pitch/Volume Analyser (PVA)}

This module analyses and outputs the frequency and its corresponding amplitude of a sound input from the Theremin at any time instance. The pitch detection in use is FFT as discussed in Section II-A. As the output of the Theremin is directly connected to the computer for analysis, we can assume the audio signal is relatively clean and hence no noise reduction/removal technique needs to be applied.

\section{B. Calibration Module}

The Calibration Module takes the joint angles of the arms and the pitch/volume information from the PVA as inputs. During the calibration phase, volume and frequency are calibrated separately.

To calibrate the pitch, we first define a neutral arm pose, e.g. a fully straightened arm. The most dominant DoF (the joint that produces the large movement of the hand) is allowed to move. We perform a search of musical notes corresponding to the joint angles. The searching pattern can be of constant, logarithmic or binary steps. Once a musical note is found, the next most dominant DoF is allowed to move away from its neutral position to explore other possible notes while keeping other DoFs fixed. This recursive process will repeat through all DoFs until all possible combinations of joint angles are found and recorded with their corresponding musical note numbers. In this work, we will only use two DoFs (the yaw DoFs of the shoulder and the elbow).
To harness the capability of aerial fingering to reach only neighbouring notes with the distal DoF, we will restrict the yaw DoF of the elbow to search for nearby notes that are immediately above and below that of the neutral position.

To calibrate the volume, we use the same approach as the pitch calibration by searching for arm positions corresponding to a list of discrete levels of desire volume. As this work does not deal with patterns of musical expressions, we only require 2 levels of volume, i.e. on and off. Thus, only 1 DoF is needed to control the volume.

\section{Feed-forward Note Player(FfNP)}

We define a vector of joint angles as $\boldsymbol{\theta}:\left[\theta_{1} \ldots \theta_{J}\right]^{T}$, where $J$ is the number of joints in use. In any case, there exists a maximum $M$ number of $\boldsymbol{\theta}_{k i}, i \in\{1 \ldots M\}$ corresponding to a musical note $p_{k}$, where $k \in\{1 \ldots N\}$ and $N$ being the maximum number of playable notes. In this work, $J$ is set to 2 while $M$ is set to 3 as described in Section III-B.

The rotational energy of a mass rotating about an arbitrary axis can be expressed as:

$$
E_{r o r}(\Delta \theta)=\tau \Delta \theta=I_{t o t} \ddot{\theta} \Delta \theta=\left(I_{c m}+m r^{2}\right) \ddot{\theta} \Delta \theta
$$

where $\tau$ is the applied torque, $I_{t o t}$ is the moment of inertia at the axis of rotation, $I_{c m}$ is the moment of inertia about an axis passing through its centre of mass, $m$ is the mass of the object and $r$ is the perpendicular distance between the two axes.

Given a melody to be played $P$ as a time-series of musical notes $\left\{p_{k}^{(x)}\right\}_{x=1}^{X}$, since every note can be played at $M$ different locations, the overall movement can simply be represented by stepping through the variable $x$ and choosing the most optimal $i$ at each $x$. We can define the cost function $C$ to derive the optimal arm position at each note as the sum of rotational energies for all the joints moved:

$$
C=\sum_{x=1}^{X} C_{x}=\sum_{x=1}^{X}\left|E_{r o r}\left(\boldsymbol{\theta}_{x}-\boldsymbol{\theta}_{x-1}\right)\right|
$$

where $x=0$ represents the initial position of the arm before playing.

Instead of evaluating all combinations of discrete energies through each note to be played, if we define $C_{i j}=\mid$ $E_{\text {ror }}\left(\boldsymbol{\theta}_{i}-\boldsymbol{\theta}_{j}\right) \mid, U$ as a possible arm position in a given $x$, $C_{x}(U)$ as the minimum energy from the starting note to the current note, we can simplify the computation into a dynamic programming problem similar to [15]:

$$
C_{x}(U)=\min _{\operatorname{arm} \operatorname{pos} v \text { in } x-1}\left\{C_{u v}+C_{x-1}(V)\right\}
$$

\section{Playing Phase}

During the playing phase, a MIDI file is loaded into the Melody Analyser for pre-processing. The Analyser ensures that all notes in the melody are within the calibrated playable range before converting the MIDI events into a time-series of commands consisting MIDI numbers and speeds. The FfNP translates the MIDI numbers into desire motor commands and sends the time-series commands to the robot. Once a particular note is played, the Next-step Error Rectifier (NsER) 
will assess the joint angles and the pitch information using the adapted non-parametric interpolation model described in Section II-B. The NsER then corrects the motor commands from the FfNP by the estimated amount needed to play the next note accurately.

\section{EXPERIMENTS}

Our Humanoid Thereminist System (Fig. 7) was implemented and evaluated on the open-source iCub robot and the Etherwave Theremin ${ }^{3}$. Three different experiments were conducted to evaluate the system quantitatively and qualitatively.

\section{A. Experimental Setup}

The configuration of the experimental setup is shown in Fig. 6. The Theremin's pitch circuit was tuned such that when the pitch antenna was held, the sound produced by the Theremin is $4.5 \mathrm{kHz}$. We constrained the hand of the iCub not to move across the plane form by the pitch antenna of the Theremin and the shoulder joint of the iCub. This was to ensure monotonic relationship between the pitch and each of the joint angles. The neutral position of the elbow yaw DoF was set at $30^{\circ}$ for calibration. We used a sampling frequency of $8 \mathrm{kHz}$ and 2048 data points for FFT analysis of pitch which gives frequency resolution of $3.91 \mathrm{~Hz}$. Since we will not deal with notes with very low frequencies, this resolution gives a sufficiently good separation between adjacent notes. One calibration was performed before all experiments to find out the range of all playable notes according to the configuration in Fig. 6. We then fixed the dials on the Theremin and the configuration for all the experiments for simpler benchmarking.

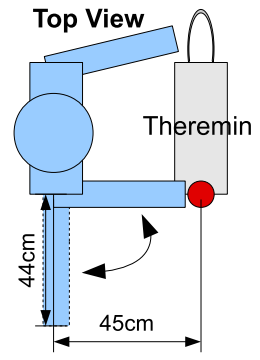

Fig. 6: Configuration of the Experimental Setup

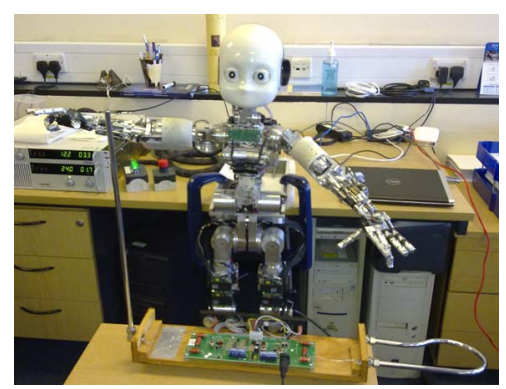

Fig. 7: The iCub Thereminist

\section{B. Experiment A: One DoF Error Margin}

As all sensors and actuators have their error margins, we implement a one DoF (shoulder yaw) Thereminist system for the pitch control to benchmark the performance of the robot dependent parameters collectively. This experiment consists of two parts. Firstly, all notes within the calibrated range are replayed in ascending sequence immediately after calibration. We then command the iCub to play two wellknown music pieces separately. The music pieces used in

\footnotetext{
${ }^{3}$ Made by Moog Music (http://www.moogmusic.com/)
}

this experiment are "Twinkle Twinkle Little Star"(Fig. 8a) and "The Entertainer"(Fig. 8b). A single channel MIDI file

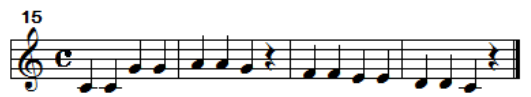

(a) Twinkle Twinkle Little Star

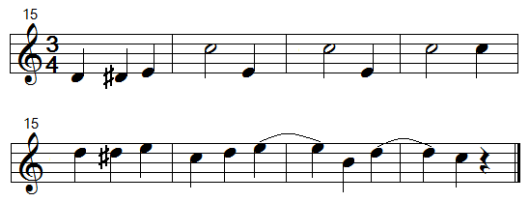

(b) The Entertainer

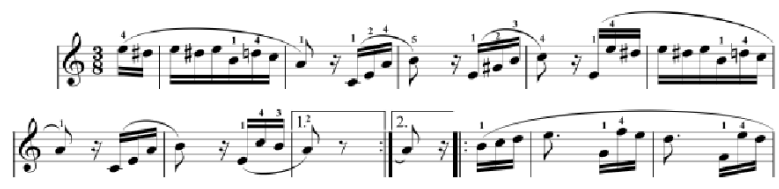

(c) Für Elise

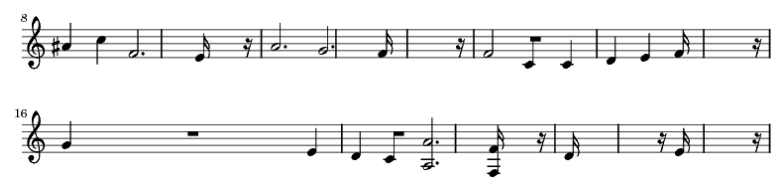

(d) Edelweiss

Fig. 8: Scores of sections of well-known music pieces.

is created for each section. The pitch information is captured and evaluated by mean absolute error (MAE) in frequency for both parts of the experiment:

$$
M A E=\frac{1}{X} \sum_{x=1}^{X}\left|f_{x}-\hat{f}_{x}\right|,
$$

where $X$ is the total number of notes played, $f_{x}$ is the ideal frequency of a note and $\hat{f}_{x}$ is the played frequency.

Since musical notes are in logarithmic scale, we should also estimate the mean percentage error (MPE) of pitch to normalise the errors across different notes:

$$
M P E=\frac{1}{X} \sum_{x=1}^{X}\left|\frac{f_{x}-\hat{f}_{x}}{f_{x}}\right|,
$$

\section{Experiment B: DoF Scaling}

To test the robustness of the System in handling more DoFs, we scale the System to 2 DoFs and allow the $2^{\text {nd }}$ DoF to have 3 alternative positions. The iCub plays the same music pieces with both 2 joints and only 1 joint activated. To benchmark the minimum energy strategy used in the FfNP Module, we set the acceleration profile of both joints to be the same so as to make $\ddot{\theta}$ in (4) constant. We then compare the energy needed to move the arms in both cases for the two pieces of music as well as the number of distinct joint movements in the 2 DoFs case. These joint movements are "shoulder only", "shoulder \& elbow" and "elbow only" in each music piece. 


\section{Experiment C: Music Playing}

In this experiment, we play a few randomly selected pieces of music using both 1 DoF and 2 DoFs for audio inspection. The musics tested are "For Alice (Für Elise)", "Edelweiss", "The Entertainer", and "Twinkle Twinkle Little Star". Extracts of these pieces are shown in Fig. 8. They are played with a range of speed to observe irregularity in rhythm. A random combination of music, speed and DoFs are used to observe any clear distinction between $1 \mathrm{DoF}$ and 2 DoFs.

\section{RESULTS AND DISCUSSIONS}

The range of playable notes was found to be between notes F4 and F6, slightly more than 2 successive Octaves which were sufficient to play all the music pieces.

\section{A. Experiment $A$}

TABLE II shows the performance comparisons of the System during calibration phase and playing phase. We extracted pitch information for musical notes that were used to play the two songs in order to benchmark their respective performance.

TABLE II: Comparison of performance between calibration phase and playing phase for two different pieces of musics

\begin{tabular}{l|c|c|c|c|c}
\hline \multirow{2}{*}{ Metrics } & \multirow{2}{*}{ Full Calib. Data } & \multicolumn{2}{|c|}{ Twinkle } & \multicolumn{2}{c}{ Entertainer } \\
\cline { 3 - 6 } & & Calib. & Actual & Calib. & Actual \\
\hline MAE (Hz) & 7.83 & 5.45 & 8.40 & 9.35 & 11.71 \\
\hline MPE & $0.86 \%$ & $0.83 \%$ & $1.25 \%$ & $1.00 \%$ & $1.12 \%$ \\
\hline
\end{tabular}

We can see that the MAEs are in the order of $1 \mathrm{~Hz}$. The largest MAE of $11.71 \mathrm{~Hz}$ shown in TABLE II, is about half of the smallest separation between two notes in the playable range. This error margin ensures all notes are distinguishable from each other. As we mentioned before, the frequency resolution we used was $3.9 \mathrm{~Hz}$, this will reduce the actual machine error further in terms of sensor data and actuation as part of the MAE is contributed by the resolution.

However, frequency resolution does not play a significant role when a note played is at a higher frequency. Thus, MPE can provide an alternative indication of the errors during both calibration and playing phases. We can see that despite the Actual MAE for playing the "Twinkle" song appears much lower than that of "Entertainer", the MPE suggests that the errors involved in playing both songs are similar. A closer look at the music scores of the songs gives an explanation for this. It is because the notes played in "Entertainer" are on average much higher than those in "Twinkle". As suggested in (3), the distance separation in arm position is proportional to note number change. This makes the frequency errors involved much larger for higher frequency notes.

The datasheet of the motors used to power the joints gives a 3\% error margin for actuation. This means that any error in actuating the joint angles that are less than $3 \%$ can be accounted for by this property of the motor. Since MPE indicates percentage change in frequency, which is proportional to music note numbers, MPE thus has a linear relationship with joint angles. Therefore, we can claim that any MPE that is smaller than 3\% (in this case, all MPEs) is within the margin of actuation error.

TABLE II also suggests that the error margin during playing phase is always bigger than that during the calibration phase by a factor less than 2. We believe that this is because larger movements were needed to play music than calibration. During playing phase, the joints are commanded to move across an arbitrary range of notes with an average of more than one note, whereas a replay of calibrated results was just a constant step of one note. This resulted in a larger absolute angle errors for music playing and hence larger error margin. The average range was calculated to be 2.5 notes per transition for "Twinkle" and 3.5 for "Entertainer". In short, we believe that all the errors can be accounted for by the error margins of the robot-dependent parameters and very little error was introduced by our Humanoid Thereminist System.

\section{B. Experiment $B$}

Fig. 9 shows the comparison of using 1 DoF and 2 DoFs in playing the two pieces of music. As we can see from both graphs, the introduction of neighbouring notes for distal DoF in a kinematic chain of joints to quickly move across, i.e. the simulation of "aerial fingering", can effectively reduce the energy needed to move the end-effector to any desired location. In fact, the graphs empirically show that the energy required to move the most dominant DoF of an arm to play any music is the upper bound of that used by any other combination of joints.

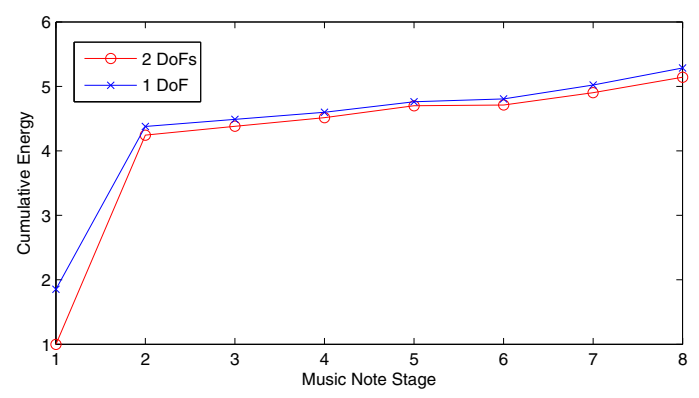

(a) Twinkle Twinkle Little Star

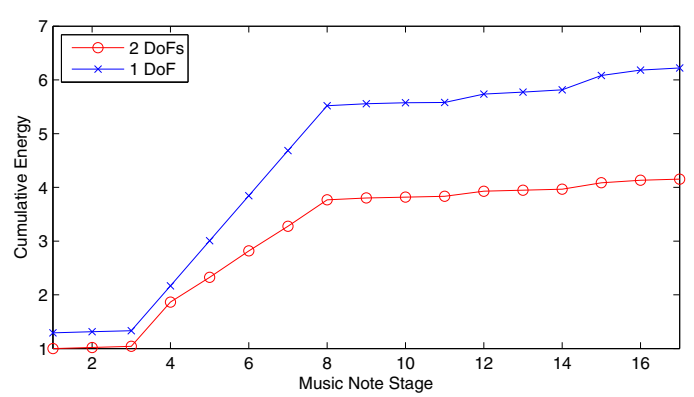

(b) The Entertainer

Fig. 9: The cumulative energy for moving the right arm of the $\mathrm{iCub}$ in playing the two music pieces using $1 \mathrm{DoF}$ and 2 DoFs approaches. The units are normalised against the smallest amount of energy in each graph.

However, the energies used in playing "Twinkle" are very 
close to each other while those used in "Entertainer" are far apart. This can be explained by the percentage usage of different types of joint movements shown in TABLE III. We can see that the use of the most dominant joint in "Twinkle" is significantly much more than the other combinations. Thus, in most music note stages, the energy used by this 2 DoFs approach is the same as the 1 DoF approach which makes the two energy graphs very similar. However, the number of elbow joint movement is comparable to the shoulder movements in "Entertainer" which implies less movement of the shoulder joint is planned by the FfNP. Since the moment of inertia about this joint is the greatest and almost twice of that of the elbow joint, this makes the energy saving much more prominent as compared to "Twinkle".

TABLE III: Percentage usage of different combination of joints in playing the two music pieces

\begin{tabular}{l|c|c|c}
\hline Music & Shoulder Only & Shoulder + Elbow & Elbow Only \\
\hline Twinkle & $75.0 \%$ & $12.5 \%$ & $12.5 \%$ \\
\hline Entertainer & $41.1 \%$ & $47.1 \%$ & $11.8 \%$ \\
\hline
\end{tabular}

Hence, we believe that our Humanoid Thereminist System scales well to an arbitrary number of DoFs with the minimum energy path planning since the worst case is the 1 DoF solution to a music piece.

\section{Experiment $C$}

In this experiment, we adapted the MIDI commands to play the music 2 times slower and faster. With random combination of musics, tempo and DoF involved, we asked 5 human subjects, all engineering postgraduate students without professional music training to see if they could differentiate the music played by the two different approaches without looking at the iCub. Although people can generally figure out notes that are occasionally off-tempo due to network bandwidth issue, no significant agreement could be drawn on which of the musics were played by a 2 DoFs arm. Thus, we believe that since the error in pitch could be explained by error margins of machine-dependent parameters, the Humanoid Thereminist System is capable of producing music of similar quality regardless of the number of DoFs are used.

\section{CONCLUSIONS}

In this paper, we proposed a two-phase Thereminist system that is capable of using multiple DoFs to play music in realtime by using a minimum energy strategy with feedback to correct pitch error for the next note stage. It consists of a calibration phase and a playing phase which can be executed independently. This System has been implemented and evaluated quantitatively and qualitatively using pitch error analysis, energy consumption and human judgement. It is also implemented to interface with MIDI file for music playing automatically. The experimental results show that our Humanoid Thereminist System is capable of produce music with reasonable amount of errors that could be justified by machine-dependent error margins. The successful deployment of multiple DoFs in playing the Theremin allows the extension of many skills, such as "aerial fingering" and musical expressions, to robotic Thereminists which require much more complex path planning mechanism.

However, as we used exhaustive search during the calibration phase, this makes the calibration process much more time-consuming when significantly more DoFs are used. We also assumed that the environment would not change drastically during both the calibration and playing phase. Although this was a reasonable assumption, we believe that further research could be conducted to look into a fast-adapting algorithm that makes the robot robust to abrupt change of environment. We also plan to look into the aspect of imitation learning for a robot to pick up Theremin-playing skills.

\section{REFERENCES}

[1] H. Longfellow, Outre-mer: a pilgrimage beyond the sea. Harper, 1835

[2] G. Weinberg and S. Driscoll, "Robot-human interaction with an anthropomorphic percussionist," in Proceedings of the SIGCHI conference on Human Factors in computing systems, pp. 1229-1232, ACM, 2006.

[3] C. Roads, "The Tsukuba musical robot," Computer music journal, vol. 10, no. 2, pp. 39-43, 1986 .

[4] J. Solis, K. Taniguchi, T. Ninomiya, T. Yamamoto, and A. Takanishi, "Development of waseda flutist robot wf-4riv: Implementation of auditory feedback system," in Proceedings of 2008 IEEE International Conference on Robotics and Automation. ICRA 2008., pp. 3654-3659, 19-23 2008.

[5] K. Shibuya, S. Matsuda, and A. Takahara, "Toward developing a violin playing robot - bowing by anthropomorphic robot arm and sound analysis," in Proceedings of the 16th IEEE International Symposium on Robot and Human Interactive Communication. RO-MAN 2007., pp. $763-768,2007$.

[6] A. Glinsky, The Theremin in the Emergence of Electronic Music. $\mathrm{PhD}$ thesis, New York University, 1992.

[7] A. Alford, S. Northrup, K. Kawamura, K. Chan, and J. Barile, "Music playing robot," in Proceedings of the 1999 International Conference on Field and Service Robotics, pp. 174-178, FSR, 1999.

[8] T. Mizumoto, H. Tsujino, T. Takahashi, T. Ogata, and H. Okuno, "Thereminist robot: Development of a robot theremin player with feedforward and feedback arm control based on a theremin's pitch model," in Proceedings of 2009 IEEE/RSJ International Conference on Intelligent Robots and Systems. IROS 2009., pp. 2297 -2302, 10 2009.

[9] R. Bellman, "Some problems in the theory of dynamic programming," Econometrica: Journal of the Econometric Society, pp. 37-48, 1954.

[10] S. Dalla Bella, I. Peretz, L. Rousseau, and N. Gosselin, "A developmental study of the affective value of tempo and mode in music," Cognition, vol. 80, no. 3, pp. B1-B10, 2001.

[11] R. Jourdain, Music, the brain, and ecstasy. Morrow, 1997.

[12] P. Welch, "The use of fast Fourier transform for the estimation of power spectra: a method based on time averaging over short, modified periodograms," IEEE Transactions on Audio and Electroacoustics, vol. 15 , no. 2 , pp. 70-73, 1967.

[13] L. Rabiner, "On the use of autocorrelation analysis for pitch detection," IEEE Transactions on Acoustics, Speech and Signal Processing, vol. 25 , pp. $24-33$, feb 1977.

[14] B. Kedem, "Spectral analysis and discrimination by zero-crossings," Proceedings of the IEEE, vol. 74, no. 11, pp. 1477-1493, 1986.

[15] Y. Wu and Y. Demiris, "Towards One Shot Learning by Imitation for Humanoid Robots," in Proceedings of 2010 IEEE International Conference on Robotics and Automation. ICRA 2010., pp. 2889-2894, May 2010. 